\title{
True Sensor Captured Radiance and Surface Reflectance from Landsat-8 Multispectral Image sets
}

\author{
C. Rajabhushanam, Allin Geo A.V, Pothumani S
}

\begin{abstract}
Proper validation of multispectral image sets that are produced by Satellite Sensors, in this era of big-data computing, is an important aspect in signal processing of natural semantic scenes. In this research article we explore fundamental issues that are cropped up in radiance correction and surface reflectance measurements. We calibrate the raw Landsat-8 imagery digital numbers by using standard functional oriented modular programs in $R$ software, for true sensor radiance and reflectance values. This important preprocessing step is essential for activities that use multispectral images for natural resources monitoring and change-detection studies. It is noted that the procedures for preprocessing can be applied in machine learning algorithms for scientific studies.
\end{abstract}

Keywords: Satellite Sensors, Algorithms, Semantic Scenes

\section{INTRODUCTION}

Traditionally, Landsat imagery from repositories such as National Remote Sensing Center[1],[3],[5], had systematic variance measures that varied from true calibrated values of the sensor. The pre-processing step of feature object retrieval was done to reduce noise effects, such as speckle, striping, salt-pepper, and irregularities in scan lines (aperiodic). To produce true sensor calibrated observed value, i.e., dampening of Quantization bit errors, the practice was to perform a viable model fit to the raw imagery, thereby reducing polarization effects[2],[4],[6].

This research work focuses on functional programming interfaces to implement processing measures that reduce abnormalities in sensor frame grabber techniques. We make sure that conversion of atmospheric radiance value is converted to true pixel reflectance DN. Thereby, improving scene capture, scene extraction, and scene modeling activities[7],[9],[11].

Specifically, generic functions that are stored as collection objects,methods that operate on inputs, model fitting, statistical calibration, and feature invariant transformation, are tested on Landsat-8 satellite imagery[14],[16],[18].

In this research article, we propose a method that produces hierarchical scale space images from input datasets that will be input to the segmentation method under deep learning

\section{Revised Manuscript Received on August 22, 2019.}

C. Rajabhushanam Assistant Professor, Department of Computer science and Engineering, Bharath Institute of Higher Education and Research, Chennai, India

Allin Geo A.V Assistant Professor, Department of Computer science and Engineering, Bharath Institute of Higher Education and Research, Chennai, India

Pothumani S Assistant Professor, Department of Computer science and Engineering, Bharath Institute of Higher Education and Research, Chennai, India framework

\section{LITERATURE REVIEW}

Landgrebe [1] describes a conceptual model that is conceived as the scene model, sensor model and the processing model. In each of the non-linear models studied, a fundamental view of the structure is in, storage and extraction of fixed variables (such as quantization, signal attenuation, and data range).[19],[21],[23] .The extraction and analysis of scene and sensor variables, is the accuracy of supervised feature methods and their parameter values to create a normalized image set. For example, Landgrebe [1], implicitly emphasizes that there is no precursor variable for data, available in the database to simulate non-lambertian forms of ground cover types. We must first simulate an environment in which scene invariant measure can be extracted by varying input parameters, such as, radiometric correction, atmospheric correction, and scene pixel normalized digital number. As the scene reflectance values do not change within band interleaving, the sensor model does not produce any variations in the results. The variations in the results are most likely from the atmospheric and geometric effects[20],[22],[24].

There are two kinds of radiometric calibration that may be used in the preprocessing steps: relative and absolute calibration. Relative method makes input data to have a sequential measurement to the next radiance value, whereas, absolute calibration makes the preprocessed data conform to an external standard, such as measures and measurements[25],[27],[29].

\section{DATA SOURCES}

Input imagery from AWS - Landsat is the primary data ingest site. While selecting a specific Path number and Row number for a defined scene, we can create a spatio-temporal fixed view of the imaging dataset. So, we will obtain a sample 3-band Landsat-8 imageset located at Chennai (India), to produce true sensor ground truth.

Using machine learning software such as $\mathrm{R}$, we will input a working algorithm for the following tasks -

To input a pseudo invariant sensing model

To input a histogram matching technique that equalizes scene differences temporally

To input a sensor radiometric correction method

To input a modifiable radiometric control set.

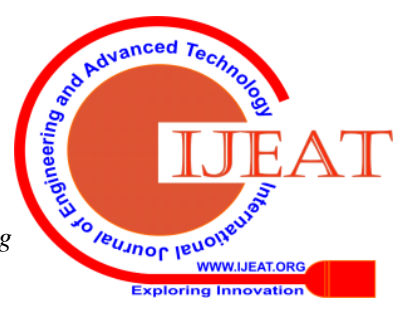


By using $\mathrm{C}$ programming language, a generic collection object that contains - Vector, Spatial range, Time, Biophysical measurements (NDVI, LAR, PAR, Correlation, Variance, and Semivariogram), will be computed along with input feature imageset[26],[28],[30].

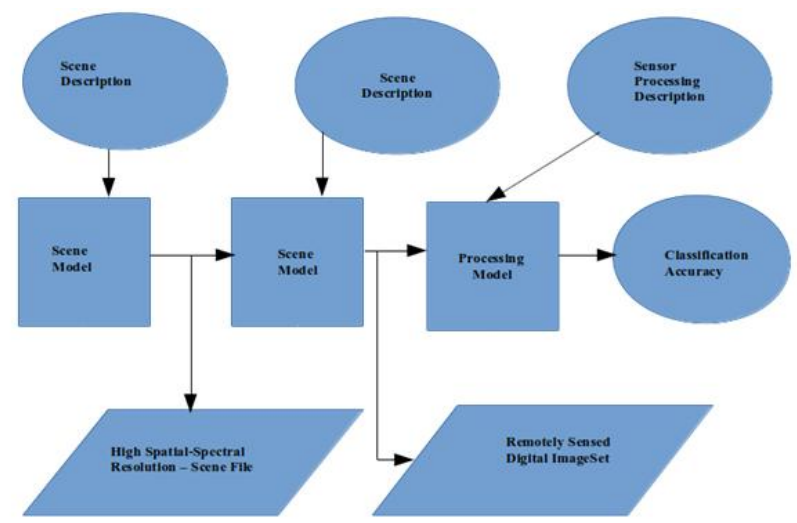

Figure 1: Scene-Sensor-Processing model for Landsat-8 Imagery

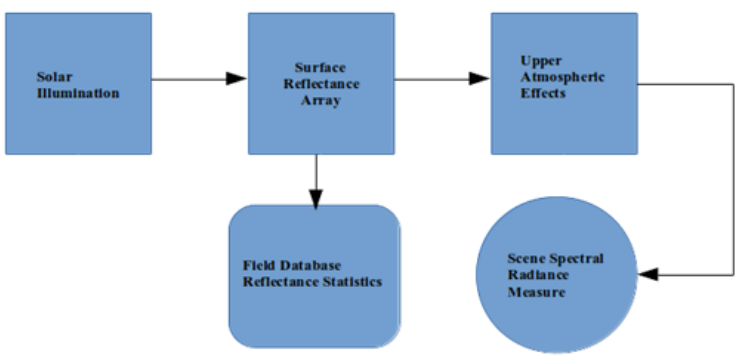

Figure 2: Sensor-Scene model of surface reflectance measure for Landsat-8 Imagery

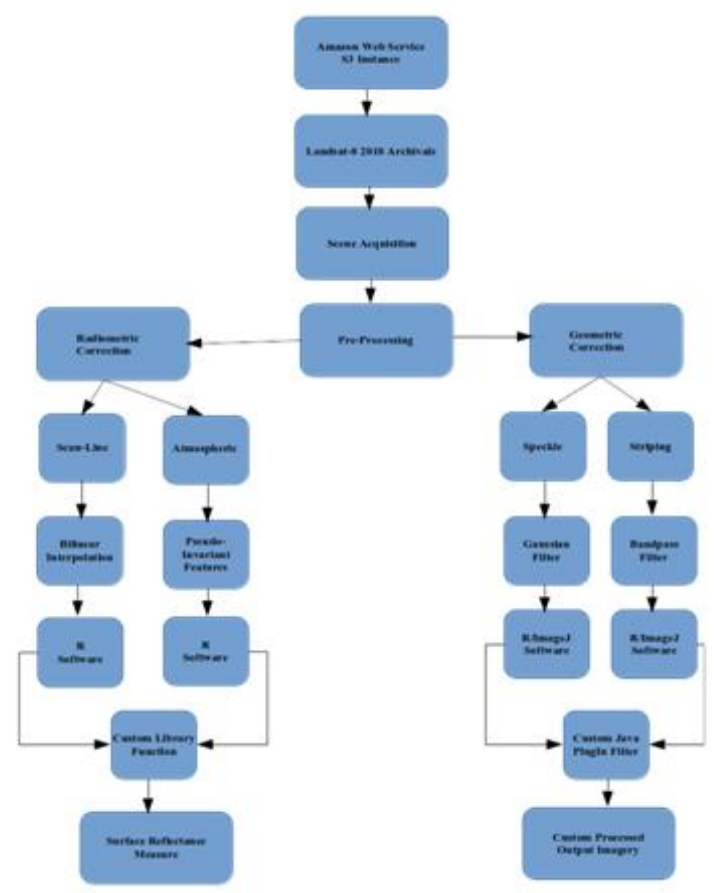

Figure 3: Research Methodology for producing true calibrated sensor

From Figure 3, it is evident that the pre-processing step involves two primary operations, namely Radiometric effects calibration and Geometric anomalies correction. Both of the operative methods can form a custom library $\mathrm{R}$ function that will inherit from the Rasterobject instance in $\mathrm{R}$.

Once we have created a virtual function that works with the two dichotomies, we can assimilate the output result objects that are modeled as a workflow routine in $\mathrm{R}$. We find that functional object-oriented structured programming in $\mathrm{R}$, enables us to implement the stated objectives, of producing true sensor surface reflectance and intensity imagery from surface radiance.

\section{RESULTS}

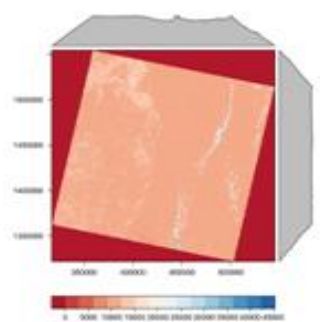

Band 3

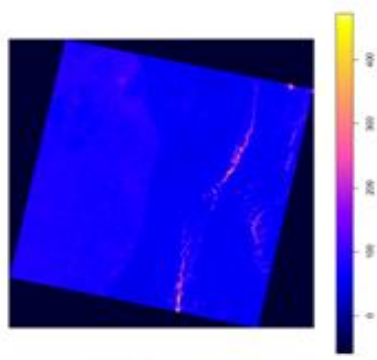

Band 3 transformed to true sensor radiance vahes

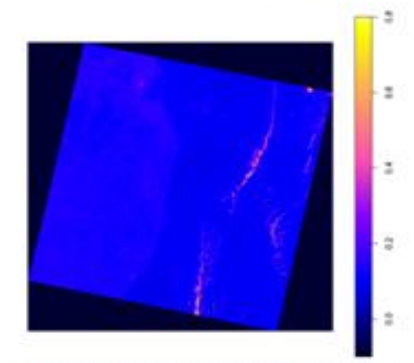

Band 3 transformed to true surface reflectance vahe

Figure4:LC08_L1TP_142051_20170410_20170414_01_T1 _B3.TIF

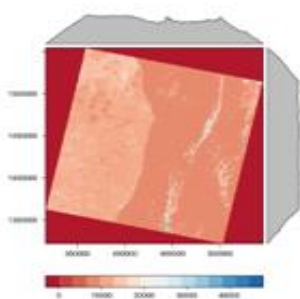

Band 4

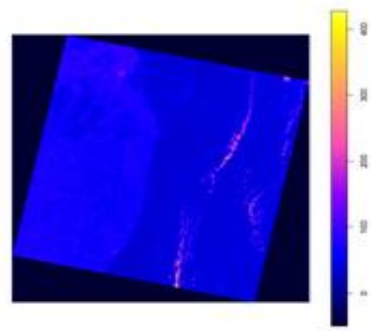

Band 4 transformed to true sensor radiance vahes

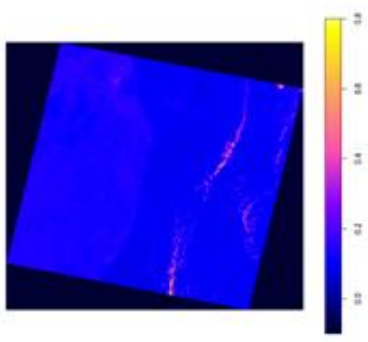

Band 4 trans formed to true surface reflectance vahes

Figure5:LC08_L1TP_142051_20170410_20170414_01_T1 _B4.TIF

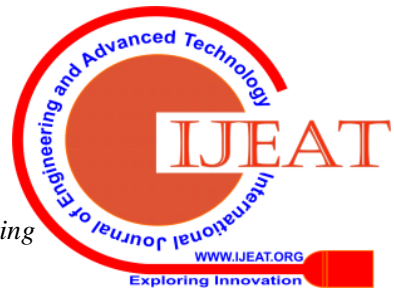



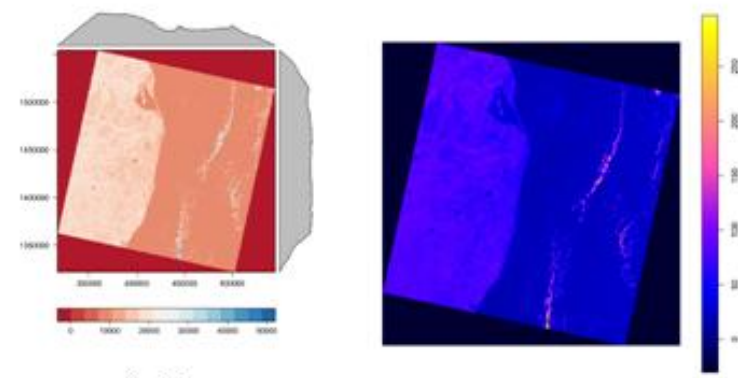

Band 5

Band 5 transformed to true sensor radiance vahues

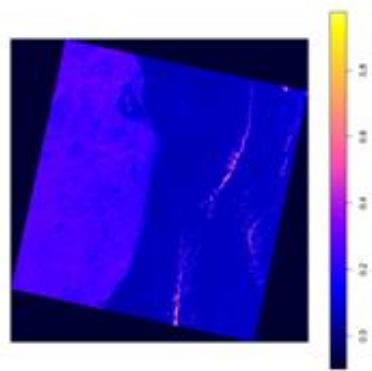

Band 5 transformed to true surface reflectance vahes

Figure6:LC08_L1TP_142051_20170410_20170414_01_T1 B5.TIF

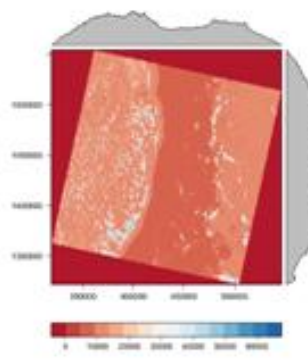

Band 3

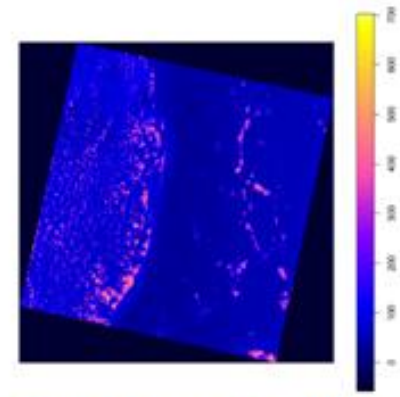

Band 3 transformed to true sens or radiance vahues

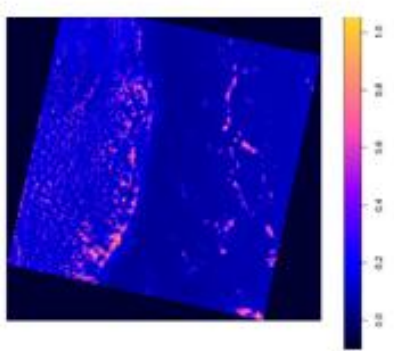

Figure7:LC08_L1TP_142051_20170512_20170525_01_T1 B3.TIF
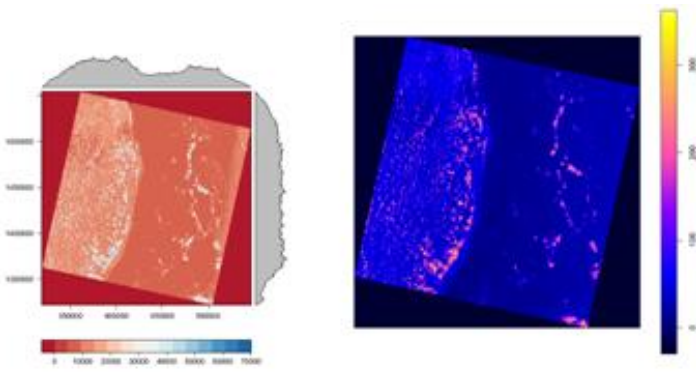

Band 4

Band 4 transformed to true sensor radiance vahues

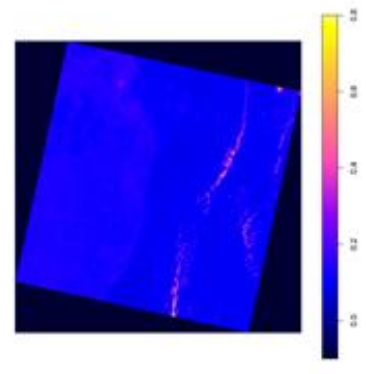

Band 4 trans formed to true surface seflectance vahes

Figure8:LC08_L1TP_142051_20170512_20170525_01_T1 B4.TIF

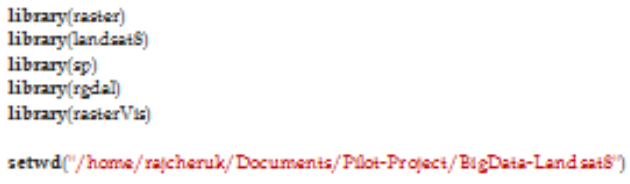

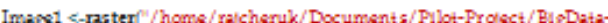
Landseis / ICOS_L1TP_142051_20170410_20170414_01_T1_B3.TIP', lever=1, valuse- TRLE') Lendents/

ngald <-2a(Image1, 'Spatia)CrdDataFrame)

rirad -radeonv(r1grid, 1.1805E-02, -59.02273 )

riref <-refle ouv(r1 grid, 20000E-05,-0.100000)

plot(nrad)

levelplot(Image1, par setting-RdBuThenes, contour-FALSE) plot(niref)

Image2 <-raster[//homs/ra/cheruk/Documents/Plbi-Profsct/BugDataLandestS/LCOS_L1TP_142051_20170410_20170414_01_T1_B4.TIP', layer=1, valuss-'TRLE') \#Imager

rigrid <-2s(Image2, 'Spatia)CndDataFranes')

rarad <-rade onv(rigrad, $9.9543 \mathrm{E}-03,-49.77129)$

Pant enteflo

plot (rend)

levelplot/Image2, parsetting-RdBuTheme, contour-FALSE)

plot(rinef)

Images <-raster ("/homs/ralcheruk/Documents/Plbot-Profsct/BugDataLandsats/LCOS_L1TP_142061_20170410_201 70414_01_T1_B5.TIP, laywr-1, values- TRLE)

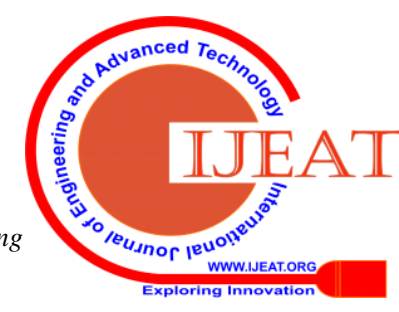


Pagrd <-2s(Images, 'SpatiaCridDataFrame')

Brad <-radcouv(13grid, 60915E-01, -30.45753)

Aref <-refle ouv(ISgrad, 20000E-05,-0.100000)

plot(Yarad)

levelplot(Inapes, par setting-RdBuThene, contour-FALSE)

plot(rsref)

Images <-raster//home/ralcheruk/Documsents/Plbot-Profect/BugData-

Landsat\$/LCOS_L1TP_142051_20170512_20170525_01_T1_B3.TIP, layer-1, valuss- TRLE

IImage

r4prid <-2s (Inages, 'Spatia) OrdDataFrame)

r4rad <-radcouv(r4grad, 1.1610E-02, -56.05023 )

ruref <-refle ouv(r4grdd, 20000E-05,-0.100000)

plot(rsad)

levelplot/Images, parsettings-RdBuTheme, contour-FALSE

plot(rursef)

Image5 <-raster["/homs/ratcheruk/ Documents/Plbi-Propect/BlgData-

LendeatS/LCOS_L1TP_142051_20170512_20170525_01_T1_B4TTP, layer=1, values- TRLE

$\#$ Image 5

rogrde <-2s(Image5, 'Spatia) CrdDataFrame')

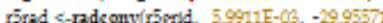

rinsef <-refleouv(n grid, 20000E- $05,-0.100000)$

plot(rorad)

levelplot(Image5, par setting-RdBuTheme, contour-FALSE)

plot(ronef)

Image6 <-raster"//home/ralcheruk/Documents/Plbi-Proloct/BugData-

Lendests/LCOS_L1TP_142051_20170512_20170525_01_T1_B5.TIP', laver=1, values- TRLE

\#Tmans

rogrid <-2s(Imagr6, 'Spatia)CndDataFrame)

rorad <-radeouv(rogreld, $1.1805 \mathrm{E}-02,-59.0227$ )

rores <-refle ouv(rogrid, 20000E-05,-0.100000)

plot(rorad)

levelplot(Inage6, parsentingr-RdBuThene, contour-FALSE)

plot(raref

R version 3.42 (2017-09-28) - "Short Summer'

Copyright (C) 2017 The R Foundation for Statistical Computin

Platform: x86_64-pc-linus-gnu (64-bit)

$R$ is free software and comes with ABSOLUTELY NO WARRANTY

You are welcome to redistribute it under certain conditions

Type 'license 0 ' or 'licence 0 ' for distribution detais

Natural language support butrunning in an English locale

$R$ is a collaborative project with many contributors

Type 'contributorso' for more information and

'citation ${ }^{\prime}$ ' on how to cite $R$ or $R$ packages in publications

Type 'demo $O$ ' for some demos, 'help $O$ for on-line help, or

'help start $O$ ' for an HAL browser interface to help.

Type ' $q O{ }^{\prime}$ to quit $R$.

$>$ library(raster)

Loading required package: sp

$>$ library(landsat8)

$>$ library(sp)

$>$ library(rgdal)

rgdal: version: 1 2-16,(SVN revision 701)

Geospatial Data A botraction Library extensions to $R$ successfully loaded

Loaded GDAL runtime: GDAL 22.1, released $2017 / 06 / 23$

Path to GDAL shared files: usr/share/gdal/2.2

GDAL binary built with GEOS: TRUE

Loaded PROJ. 4 runtime: Rel 493,15 August2016, [PJ_VERSION: 493]

Path to PROJ.4 shared files: (autodetected)

Linking to $\mathrm{sp}$ version: $12-$ ?

$>$ library(rasterVis)

Loading required package: lattice

Loading required package: latticeExtra

Loading required package: RColorBrewer

> setwd(" /home rajcheruk Documents / Pilot-Project BigData-Landsat8")

$>\operatorname{sink}$ (file $=$ "/home rajcheruk Documents Pilot-Project BigData-Landsat8 Radiance_rm log" append $=$ RUE, type $=c$ ("output", "message"), split = FALSE

$>$ pdf("/home rajcheruk Documents Pilot-Project BigData-Landsat8 PixelRadiance pdf" useDingbats = FALSE
>Imagel <-raster("/home rajcheruk/ Documents Pilot-Project BigData-

Landsat8/LC08_L1TP_142051_20170410_20170414_01_T1_B3.TIF", layer=1 values $=$ " TRUE"

$>=$ Imagel

$>$ rl prid <- as (Imagel, 'SpatialGridDataFrame')

$>$ rl rad <-radconv(rl grid, $1.1805 \mathrm{E}-02,-59.02273$ )

$>$ rlref<-reflconv(rl grid, $2.0000 \mathrm{E}-05,-0.100000$ )

$>$ plot(rlrad)

$>$ levelplot(Imagel, par settings=RdBuTheme, contour=FALSE)

$>$ plot(rlref)

$>$ Image2 <-raster("/home rajcheruk Documents Pilot-Project BigData-

Landsat8/LC08_L1TP_142051_20170410_20170414_01_T1_B4.TF", layer=1, values $=$ "TRUE"

$>=$ Inage 2

$>12$ grid $<-$ as(Image2, 'SpatialGridDataFrame')

$>12$ rad <- radconv( $(2$ grid, $99543 \mathrm{E}-03,-49.77129)$

$>$ r ref <- reflconv(rl grid, $2.0000 \mathrm{E}-05,-0.100000)$

$>$ plot $(\mathrm{r} 2 \mathrm{rad})$

$>$ levelplot(Image2, par settings=RdBuTheme, contour=FALSE)

$>$ plot(rref)

$>$ Image 3 <-raster("/home rajcherulk Documents Pilot-Project BigData-

$>$ Image 3 <-raster("/home rajcherule Documents Pilot-Project BigData-
Landsat8/LC08_L1TP_142051_20170410_20170414_01_T1_B5.TF", layer=1 values $=$ " $\left(\right.$ RUE $^{n}$ )

$>=$ Image 3

$>13$ grid <- as(Image3, 'SpatialGridDataFrame')

$>\mathrm{r} 3 \mathrm{rad}<-$ radconv(r3 grid, $6.0915 \mathrm{E}-03,-30.45755)$

$>$ r 3 ref $<$ - reflconv(r3 grid, $2.0000 \mathrm{E}-05,-0.100000)$

$>$ plot(r3rad)

$>$ levelplot(Image 3 , par settings=RdBuTheme, contour=FALSE)

$>$ plot(r3ref)

$>$ Image4 <-raster("/home rajcheruk Documents Pilot-Project BigData-

Landsat8/LC08_L1TP_142051_20170512_20170525_01_T1_B3.TIF",layer=1. values $=$ 'TRUE $\overline{ }$ )

$>=$ Image 4

$>14$ grid <- as(Image4, 'SpatialGridDataFrame')

$>$ r4rad <-radconv(r4 grid, 1.1610E-02,-58.05023)

$>14 r e f<-r e f l c o n v(r 4$ grid, $2.0000 \mathrm{E}-05,-0.100000)$

$>$ plot( $(\mathrm{r} 4 \mathrm{rad})$

$>$ levelplot(Inage4, par settings=RdBuTheme, contour=FALSE)

$>$ plot(r4ref)

$>$ Image5 <-raster("/home raj cheruk/ Documents Pilot-Project BigDat

Landsat8/LC08_L1TP_142051_20170512_20170525_01_T1_B4.TIF", layer=1

values $=$ 'TRUE")

$>=$ Image 5

$>$ r5 grid <-as(Image5, 'SpatialGridDataFrame')

$>\mathrm{r} 5 \mathrm{rad}<-\mathrm{radconv}(\mathrm{r} 5$ grid, $59911 \mathrm{E}-03,-29.95571)$

$>$ r5ref<-reflconv(rl grid, $2.0000 \mathrm{E}-05,-0.100000$ )

$>$ plot(r5 rad)

$>$ levelplot(Image5, par settings=RdBuTheme, contour=FAL SE)

$>$ plot(r5ref)

$>$ Image6 <-raster("/home rajcheruk/ Documents Pilot-Project BigData-

Landsat8/LC08_L1TP_142051_20170512_20170525_01_T1_B5.MI", layer=1.

values $=$ 'TRUE"

$>=$ Image 6

$>16$ grid <-as(Image6, 'SpatialGridDataFrame')

$>$ r6rad <-radconv(r6 grid, $1.1805 \mathrm{E}-02,-59.02273$ )

$>$ r6ref <-reflconv(r6 grid, $2.0000 \mathrm{E}-05,-0.100000)$

$>$ plot(r6rad)

$>$ levelplot(Image6, par settings=RdBuTheme, contour=FALSE)

$>$ plot(roref)

$>$ proctime 0

user system elapsed

28286562082662973

\section{REFERENCES}

1. Gowri Sankaran, B., Karthik, B. \& Vijayaragavan, S.P. 2019, "Weight ward change region plummeting change for square based image huffman coding", International Journal of Innovative Technology and Exploring Engineering, vol. 8, no. 10, pp. 4313-4316.

2. Gowri Sankaran, B., Karthik, B. \& Vijayaragavan, S.P. 2019, "Image compression utilizing wavelet transform", International Journal of Innovative Technology and Exploring Engineering, vol. 8, no. 10, pp. 4305-4308.

3. Kandavel, N. \& Kumaravel, A. 2019, "Offloading computation for efficient energy in mobile cloud computing", International Journal of Innovative Technology and Exploring Engineering, vol. 8, no. 10 , pp. $4317-4320$

Published By

Blue Eyes Intelligence Engineering \& Sciences Publication

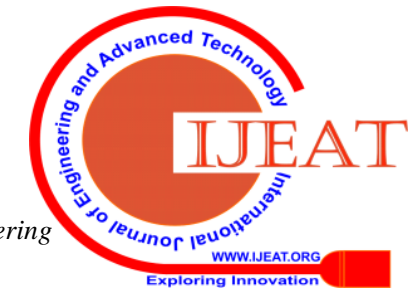


4. Vinoth, V.V. \& Kanniga, E. 2019, "Reversible data hiding in encrypting images-an system", International Journal of Engineering and Advanced Technology, vol. 8, no. 6, pp. 3051-3053.

5. Selvapriya, B. \& Raghu, B. 2019, "Pseudocoloring of medical images: A research", International Journal of Engineering and Advanced Technology, vol. 8, no. 6, pp. 3712-3716.

6. Senthil Kumar, K. \& Muthukumaravel, A. 2019, "Bi-objective constraint and hybrid optimizer for the test case prioritization", International Journal of Engineering and Advanced Technology, vol. 8, no. 6 , pp. $3436-3448$.

7. Kavitha, G., Priya, N., Anuradha, C. \& Pothumani, S. 2019, "Read-write, peer-to-peer algorithms for the location-identity split", International Journal of Innovative Technology and Exploring Engineering, vol. 8, no. 9 Special Issue 3, pp. 445-447.

8. Kaliyamurthie, K.P., Michael, G., Anuratha, C. \& Sundaraj, B. 2019, "Certain improvements in alzheimer disease classification using novel fuzzy c means clustering for image segmentation", International Journal of Innovative Technology and Exploring Engineering, vol. 8, no. 9 Special Issue 3, pp. 599-604.

9. Kaliyamurthie, K.P., Sundarraj, B., Geo, A.V.A. \& Michael, G. 2019, "RIB: Analysis of I/O automata", International Journal of Innovative Technology and Exploring Engineering, vol. 8, no. 9 Special Issue 3, pp. 1019-1022.

10. Velvizhi, R., Rajabhushanam, C. \& Vidhya, S.R.S. 2019, "Opinion mining for travel route recommendation using Social Media Networks (Twitter)", International Journal of Innovative Technology and Exploring Engineering, vol. 8, no. 9 Special Issue 3, pp. 508-512.

11. Kavitha, R., Sangeetha, S. \& Varghese, A.G. 2019, "Human activity patterns in big data for healthcare applications", International Journal of Innovative Technology and Exploring Engineering, vol. 8, no. 9 Special Issue 3, pp. 1101-1103.

12. Pothumani, S., Anandam, A.K., Sharma, N. \& Franklin, S. 2019, "Extended VEOT framework - Implemented in a smart boutique", International Journal of Innovative Technology and Exploring Engineering, vol. 8, no. 9 Special Issue 3, pp. 762-767.

13. Kaliyamurthie, K.P., Michael, G., Krishnan, R.M.V. \& Sundarraj, B. 2019, "Pseudorandom techniques for the internet", International Journal of Innovative Technology and Exploring Engineering, vol. 8, no. 9 Special Issue 3, pp. 915-918.

14. Aravindasamy, R., Jeffrin Rajan, M., Rama, A. \& Kavitha, P. 2019 "Deep learning provisions in the matlab: Focus on CNN facility", International Journal of Innovative Technology and Exploring Engineering, vol. 8, no. 9 Special Issue 3, pp. 990-994.

15. Theivasigamani, S., Linda, M. \& Amudha, S. 2019, "Object sensing and its identification \& motion sensing", International Journal of Innovative Technology and Exploring Engineering, vol. 8, no. 9 Special Issue 3, pp. 545-549.

16. Mary Linda, I., Vimala, D. \& Shanmuga Priya, K. 2019, "A methodology for the emulation of IPv4", International Journal of Innovative Technology and Exploring Engineering, vol. 8, no. 9 Special Issue 3, pp. 848-852.

17. Velvizhi, R., Priya, D.J., Vimala, D. \& Linda, I.M. 2019, "Increased routing algorithm for mobile adhoc networks", International Journal of Innovative Technology and Exploring Engineering, vol. 8, no. 9 Special Issue 3, pp. 1606-1608.

18. Sangeetha, S., Anuradha, C. \& Priya, N. 2019, "DNS in real world", International Journal of Innovative Technology and Exploring Engineering, vol. 8, no. 9 Special Issue 3, pp. 937-940.

19. Geetha, C., Vimala, D. \& Priya, K.S. 2019, "Constructing multi-processors and spreadsheets with SKIVE", International Journal of Innovative Technology and Exploring Engineering, vol. 8, no. 9 Special Issue 3, pp. 516-519.

20. Yugendhar, K., Sugumar, V. \& Kavitha, P. 2019, "A novel method of univac using fuzzy logic", International Journal of Innovative Technology and Exploring Engineering, vol. 8, no. 9 Special Issue 3, pp. 435-437.

21. Kaliyamurthie, K.P., Michael, G., Elankavi, R. \& Jijo, S.A. 2019, "Implementing aggregate-key for sharing data in cloud environment using cryptographic encryption", International Journal of Innovative Technology and Exploring Engineering, vol. 8, no. 9 Special Issue 3, pp. 957-959.

22. Jeffrin Rajan, M., Aravindasamy, R., Kavitha, P. \& Rama, A. 2019, "A novel method of object orientation variation in $\mathrm{C}++$ and java", International Journal of Innovative Technology and Exploring Engineering, vol. 8, no. 9 Special Issue 3, pp. 708-710.
23. Nayak, R., Dinesh, S. \& Thirunavukkarasu, S. 2019, "A novel method improvement of rapid miner for the data mining applications", International Journal of Innovative Technology and Exploring Engineering, vol. 8, no. 9 Special Issue 3, pp. 457-460.

24. Sivaraman, K., Krishnan, R.M.V., Sundarraj, B. \& Sri Gowthem, S. 2019, "Network failure detection and diagnosis by analyzing syslog and SNS data: Applying big data analysis to network operations", International Journal of Innovative Technology and Exploring Engineering, vol. 8, no. 9 Special Issue 3, pp. 883-887.

25. Vimala, D., Linda, I.M. \& Priya, K.S. 2019, "Decoupling online algorithms from erasure coding in DNS", International Journal of Innovative Technology and Exploring Engineering, vol. 8, no. 9 Special Issue 3, pp. 950-953.

26. Rama, A., Kumaravel, A. \& Nalini, C. 2019, "Preprocessing medical images for classification using deep learning techniques", International Journal of Innovative Technology and Exploring Engineering, vol. 8 , no. 9 Special Issue 3, pp. 711-716.

27. Sangeetha, S., Srividhya, S.R., Anita Davamani, K. \& Amudha, S. 2019, "A procedure for avoid overrun error in universal synchronous asynchronous receiver transmitter (usart) by utilizing dummy join and interrupt latency method", International Journal of Innovative Technology and Exploring Engineering, vol. 8, no. 9 Special Issue 3, pp. 657-660.

28. Aravindasamy, R., Jeyapriya, D., Sundarajan, B. \& Sangeetha, S. 2019, "Data duplication in cloud for optimal performance and security", International Journal of Innovative Technology and Exploring Engineering, vol. 8, no. 9 Special Issue 3, pp. 1156-1158.

29. Aravindasamy, R., Jeffrin Rajan, M., Sugumar, V. \& Kavitha, P. 2019, "A novel method on developing superblocks and the transistor using apodryal", International Journal of Innovative Technology and Exploring Engineering, vol. 8, no. 9 Special Issue 3, pp. 982-985.

30. Sasikumar, C.S. \& Kumaravel, A. 2019, "E-learning attributes selection through rough set theory and data mining", International Journal of Innovative Technology and Exploring Engineering, vol. 8 , no. 10 , pp. $3920-3924$

\section{AUTHORS PROFILE}

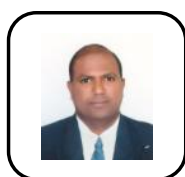

C. Rajabhushanam Assistant Professor, Department of Computer science and Engineering, Bharath Institute of Higher Education and Research, Chennai, India

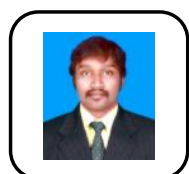

Allin Geo A.V Assistant Professor, Department of Computer science and Engineering, Bharath Institute of Higher Education and Research, Chennai, India

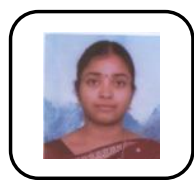

Pothumani S Assistant Professor, Department of Computer science and Engineering, Bharath Institute of Higher Education and Research, Chennai, India 